\title{
The relationship between self-control and procrastination among adolescent: The mediating role of multi screen addiction
}

\author{
Zeynep Şimşir Gökalp ${ }^{1}$ (D) Mustafa Saritepeci² ${ }^{2} \cdot$ Hatice Yildiz Durak ${ }^{3}$ (i)
}

Accepted: 1 November 2021

(c) The Author(s), under exclusive licence to Springer Science+Business Media, LLC, part of Springer Nature 2021

\begin{abstract}
Self-control is always crucial in many areas of life. Therefore, self-control failure is the source of many of the difficulties people face in their lives and also at the center of several problems, especially among adolescents. In this regard, the purpose of the study was to examine the mediating role of multi-screen addiction (MSA) in the relationship between self-control and procrastination among adolescents by using structural equation modeling (SEM). A cross-sectional design and an online questionnaire was used in this study. The study group composed of 390 adolescents studying at various high schools in Turkey. The results of correlation analysis showed that self-control was negatively correlated with MSA and procrastination. MSA also positively correlated with procrastination. Furthermore, the findings showed that MSA mediated the relationship between self-control and procrastination. The fit index of the SEM was found to be satisfactory. The results of the study were addressed in the context of the existing literature, and then suggestions were presented.
\end{abstract}

Keywords Self-control $\cdot$ Multi-screen addiction $\cdot$ Procrastination $\cdot$ Adolescents $\cdot$ Path analysis

Self-control is often conceptualized as the capacity to suppress or override immediate desires to achieve a long-term objective (Baumeister et al., 2007; Metcalfe \& Mischel, 1999; Tangney et al., 2004). Self-control, as willpower in general and executive function in particular, is considered to refer to mental functions that help people resist desires, manage competing tasks, and maintain attention. (Inzlicht et al., 2014). Individuals with greater self-control have more control of their thoughts, feelings, and urges than people with poor self-control (Baumeister et al., 1998). Therefore,

Zeynep Şimşir Gökalp

zey.simsir.93@gmail.com

Mustafa Saritepeci

mustafasaritepeci@gmail.com

Hatice Yildiz Durak

hatyil05@gmail.com

1 Faculty of Education, Department of Guidance and Psychological Counseling, Selcuk University, Selcuk, Turkey

2 Faculty of Eregli Education, Department of Computer Education and Instructional Technology, Necmettin Erbakan University, Konya, Turkey

3 Faculty of Science, Department of Computer Technology and Information Systems, Bartın University, Bartin, Turkey self-control is essential for success in most aspects of life, particularly education, employment, and relationships (Inzlicht et al., 2014; de Ridder et al., 2012). A growing body of research demonstrated that high self-control is related to adaptive behaviors such as success work/school achievement, health-related behaviors, interpersonal success, less pathology, adjustment, life satisfaction, and well-being (de Ridder et al., 2012; Duckworth \& Seligman, 2005; Ronen et al., 2016; Şimşir \& Dilmaç, 2021; Tangney et al., 2004). Consistent with these results, a low level of self-control was related to maladaptive behavior such as criminality, drug addictions, behavioral addictions (e.g., smartphone, internet, social media), binge eating, bullying behaviors, and procrastination (de Ridder et al., 2012; Geng et al., 2018; Grasmick et al., 1993; Vainik et al., 2019).

Procrastination, one of the consequences of a failure of self-control (Steel, 2007), is characterized as the deliberate postponement of a planned, required, and/or personally significant action, despite the likelihood of negative outcomes outweighing the potential benefits of the delay (Klingsieck, 2013). Procrastination, which greatly troubled individuals and society, is extremely common (Steel, 2007; Zhang et al., 2019). Many research showed that procrastination is linked to a variety of negative effects, particularly among adolescents (e.g., Kadzikowska-Wrzosek, 2020; Kim \& Seo, 2015; 
Klassen et al., 2009). Hence, it is substantial to explore the mechanism behind procrastination.

Sirois and Pychyl (2013) suggested that selecting to intentionally delay despite intention indicates a fundamental breakdown in self-control/regulation. This breakdown happens more often, when individuals confront with an undesired task (i.e., stressful, boring, missing sense and/or structure), resulting in negative emotions or a low mood. For example, if a task is seen as challenging, tedious, or unpleasant, people try to avoid it (Seneecal et al., 1997). On the other hand, the internet offers numerous enjoyable distractions and is often characterized as a means of obtaining a fun, interesting, and entertaining experience that helps relieve perceived stress (Lavoie \& Pychyl, 2001). Similarly, screen devices (computer, tablet, smartphone, TV) provide gratification to eliminate leisure boredom. As a result, while this can help people to meet their sensation-seeking needs, it also raises the likelihood of screen addiction (Lin et al., 2019). In the end, screen dependent people are unable to suppress their desires and may spend more time in front of the screen, resulting in increased procrastination.

Screen addiction refers to a problematic and dependent attitude to a range of screen activities (Balhara et al., 2018). Internet addiction (gambling, buying, sexuality, general), digital game addiction (online, offline, single-player, multiplayer, depending on the genre), media addiction (traditional media, social media), and technical device addiction (computers, television, smartphones, tablet, VR and so on.) are all regarded to be subtypes of screen addiction. Digital contact, virtual environment, and screen are three dynamics that are frequent in these subtypes and can lead to addiction on their own or in combination (Macit \& Kavafoğlu, 2019). Consequently, multi-screen addiction (MSA) like smartphone addiction or internet addiction is regarded as a behavioral addiction. Because people who use several screens tend to lose control and develop behavioral addiction (Saritepeci, 2021).

For this purpose, in light of the aforementioned explanations, the main aim of the study was to examine associations between self-control, MSA, and procrastination by using a structural equation model (SEM).

\section{The Importance of the Study}

Screen addiction creates harmful addiction results in terms of social, health, and behavioral problems (Balhara et al., 2018; Chang et al., 2018; Lin et al., 2019; Monacis et al., 2021). This topic attracted attention in studies on humanmachine interactions that are addictive because of the numerous negative impacts that users' excessive screen time may have on themselves and others. However, few studies addressed screen addiction, especially in the adolescent population. Previous studies reported that behavioral addictions such as smartphones (Haug et al., 2015), the internet (Tang et al., 2014), video, and internet gaming (Wang et al., 2014) are very prevalent among adolescents. The results of these researches are probably limited to the devices or platforms under investigation, and the views given by such research are thus restricted in terms of representing the multi-screen context. Since screen addiction covers all addiction types, addressing screen addiction in research will present more comprehensive results.

Adolescents' ability to exercise self-control has great importance. The adolescent years are characterized by a variety of social and biological difficulties (Crone \& Dahl, 2012). Lack of self-control may hamper adolescents' ability to tackle these difficulties (Willems et al., 2018). Furthermore, since self-control capacity during childhood and adolescence influences later life outcomes, it is thought to be especially essential during this period (Allemand et al., 2019). For this reason, it is important to examine the selfcontrol behavior of adolescents and their behaviors related to self-control. The current study will give preliminary information to the link between self-control, MSA, and procrastination in adolescents as it aims to explore the correlations between these variables. In addition, it will contribute to the literature since it includes self-control measures for preventing MSC and procrastination in adolescents.

The theoretical foundation of the present study was built on a strength model of self-control known as ego depletion theory (Baumeister et al., 1998; Baumeister et al., 2007; Muraven \& Baumeister, 2000). In the existing literature, this theory has received a great deal of empirical consideration and support (Hagger et al., 2010). However, much research has mostly concentrated on short-term self-control activities and stages of rest in lab conditions (e.g., Baumeister et al., 1998; Muraven \& Baumeister, 2000). This study, which uses a different method (relational approach) will make a significant contribution to the development of the theory.

\section{Conceptual Framework}

\section{Theoretical Background}

Various theories provide a framework for understanding selfcontrol behavior. For example, A General Theory of Crime emphasizes low self-control explaining the cause of criminal acts (Gottfredson \& Hirschi, 1990). The concept of "low self-control" is used by Gottfredson and Hirschi to characterize a persistent "criminality" or "criminal inclination" that increases the likelihood that people will not be able to resist the simple, instant gratification of crime (Pratt \& Cullen, 2000). Besides, individuals' level of self-control has an impact on their performance or results in environments 
like school, the workplace, and relationships (Gottfredson \& Hirschi, 1990). On the other hand, the Hot/Cool System Approach was developed relying on the delay of gratification perspective to understand the mechanism of self-control or willpower (Metcalfe \& Mischel, 1999; Mischel \& Ebbesen, 1970). Self-control is seen as a component of the cool-cognitive system, which directs goal-directed actions and depends on people's volitional control to perform. The hot system is connected with poor self-control and the tendency to impulsive behavior since it functions on emotional principles. The combination of these systems enables self-control behavior to be understood (Metcalfe \& Mischel, 1999).

This study's theoretical framework is established on the Strength Model of Self-Control (Baumeister et al., 1998). According to this model, self-control requires the use of a finite resource or energy. Part of the resource will be consumed during the initial act of self-control. Afterward, the second self-control goal would be impaired and difficult to complete. Self-control is depleted after use, just as a muscle gets tired, losing some of its strength, but also, regular practice helps to strengthen it. Furthermore, the model and empirical reports revealed that various forms of self-control (e.g., compulsion resistance, impulse control, emotion regulation, continuity in a task, and decision making) depend on a common, finite resource. The key point is that exercising self-control consumes resources required for a wide range of self-regulatory actions in several behavioral areas, increasing the likelihood of subsequent self-control breakdown (de Ridder et al., 2012; Hagger et al., 2010). In the end, ego depletion caused by acts of self-control will make people more passive, interfering with subsequent behavior (Baumeister et al., 1998). In this regard, if someone does not have adequate psychological resources and motivation to control him/herself when starting a task, he/she would postpone doing it (Geng et al., 2018; Kamphorst et al., 2018).

\section{Theoretical Relationship Between the Variables}

As indicated earlier, procrastination refers to the deliberate postponement of a future task despite the likelihood of being harmed as a result of the delay (Steel, 2007). Procrastination could be characterized by a lack of self-control (Rebetez et al., 2016). Individuals with low self-control tend to procrastinate because they have few resources (energy) to devote to work or study (Zhao et al., 2019). On the other hand, a high level of willpower compensates for the use of psychological resources, thus decreasing procrastination (Job et al., 2010).

Additionally, according to the strength model of selfcontrol, people who reduced energy may tend towards activities that they can easily obtain and spend less energy (Baumeister \& Alquist, 2009; Hofmann et al., 2017; Reinecke et al., 2014). Screen devices are the most available alternative (Lin et al., 2019; Saritepeci, 2021) for procrastinators these days. Moreover, behaviors that become habitual by being automated don't consume much energy (Baumeister \& Tierney, 2011; de Ridder et al., 2012; Hofmann et al., 2017). Baumeister and Alquist (2009) emphasized that habits, whether desired or undesired, are more resistant to depletion because they require less energy. In this context, previous research has also demonstrated a correlation between perceived cognitive resource depletion and the use of media as a procrastination mechanism. A study by Reinecke et al. (2014) reported that low situational self-control ability (also known as ego depletion) increased participants' perceptions of procrastinating after work TV and video games. Therefore, it is an expected result for people with screen addiction to tend to these devices and display procrastination behavior. Apparently, the relationship between self-control and procrastination and the role of MSA can be explained with the strength model of self-control.

\section{Self-Control and Procrastination}

Considering the theories explaining self-control, it can be seen that self-control consists of two elements that inhibit and initiate behavior (de Ridder et al., 2011; de Ridder et al., 2012). High trait self-control is commonly characterized by a strong ability to resist temptation, stressing self-control as an inhibitory mechanism. Effective self-control refers to overcoming impulses and behaviors that can lead to problems such as smoking, drinking, taking drugs, aggression, cheating, or unsafe sex (Ent et al., 2015). People who execute self-control in daily life do not eat or drink what they want, do not buy what they like, do not have sex with others they want, do not strike or kill others they dislike and do a variety of other things to satisfy their desires (Baumeister \& Alquist, 2009). In this context, the understanding of procrastination as a failure of self-control proposes that the inability to carry out the previous intention, resulting in delay, is largely due to the failure of inhibition capacities (Steel, 2007).

As stated earlier, people with low self-control scores are not able to resist impulses frequently and successfully (Baumeister et al., 1998; Ent et al., 2015; Tangney et al., 2004). Similarly, procrastinators have difficulty resisting their impulses (Ferrari \& Emmons, 1995; Zhang et al., 2019). Ferrari and Emmons (1995) also emphasized that individuals could procrastinate since they can't control their impulses for pleasurable, immediate activities at the cost of completing essential tasks. Additionally, the previous studies have explicitly demonstrated the link between self-control and procrastination (Ferrari \& Emmons, 1995; Geng et al., 2018; Kim et al., 2017; Steel, 2007; Zhao et al., 2019). However, this link has received little attention among adolescents (e.g., Kuhnle et al., 2011). Taken all together, this study expected procrastination may exist in the presence of low 
self-control among adolescents. In this regard, the relevant research hypothesis was presented below:

H1. There is a negative relationship between self-control and procrastination among adolescents.

\section{Self-Control and MSA}

Over the last decade, there has been a significant increase in studies into "addictive technological behaviors" (Andreassen et al., 2016). Since users have become addicted to technological devices and networks as a result of their excessive use, this has become a significant source of concern (Andreassen, 2015; Andreassen et al., 2016). Significantly, adolescents are at risk in terms of internet addiction (Tang et al., 2014), problematic social media usage (Yıldız Durak, 2020), and smartphone addiction (Saritepeci, 2020). Additionally, such addictions create serious problems in terms of adolescents' physical and mental health as in other addiction types (i.e., drug, cigarette) (Andreassen et al., 2016; Reinecke et al., 2018; Tang et al., 2014). In this context, in section 3 of the Diagnostic and Statistical Manual of Mental Disorders (DSM-5), Internet Gaming Disorder was identified as a possible behavioral addiction (American Psychiatric Association, 2013). For this reason, adolescents' behaviors related to technological addictions should be handled with a more detailed and comprehensive approach.

Screen addiction is characterized as a range of uncontrolled media habits involving various screen devices (personal computers, laptops, tablets, and smartphones), ranging from compulsive media use to highly unhealthy and even addictive behaviors (Lin et al., 2019). In this sense, Hofmann et al. (2017) emphasized the importance of self-control in getting a greater understanding of the dynamic relationships between media use and well-being. One of the main reasons behind addictive technology use such as internet addiction (Kim et al., 2017), problematic mobile use (Çebi et al., 2019), online gaming addiction (Mehroof \& Griffiths, 2010), and problematic social media usage (Y1ldız Durak, 2020) is the failure of self-control. As a result, inadequate self-control may trigger MSA. However, there is still a lack of studies on the link between adolescents' self-control levels and MSA behaviors. In this regard, the relevant research hypothesis is below:

H2. There is a negative relationship between self-control and MSA among adolescents.

\section{MSA and Procrastination}

Procrastination refers to the habit of putting off tasks that must be completed (Ferrari \& Emmons, 1995). Numerous research has been carried out to understand why individuals procrastinate and to investigate the neurological substrates of procrastination (Steel, 2007; Zhang et al., 2019). Five common traits that cause procrastination were identified as task aversiveness, potential rewards, the time delay of these rewards, self-control, and impulsivity (Zhang et al., 2019). On the other hand, procrastination may occur when someone engages in behaviors that they enjoy, such as watching entertaining television, while deliberately deferring the execution of other (more important or demanding) tasks (Lavoie \& Pychyl, 2001; Reinecke et al., 2014). This is consistent with Geng and colleague's (Geng et al., 2018) findings, which indicated that procrastinators postponed more of their plans mainly due to the enjoyable options (e.g., internet), which was interpreted as a lack of desire to resist temptations during planned activities. A large body of research also demonstrated that media use (Lavoie \& Pychyl, 2001; Reinecke et al., 2014; Reinecke \& Hofmann, 2016), internet use (Reinecke et al., 2018; Yang et al., 2019), and mobile devices (Çebi et al., 2019) are all common ways to procrastinate. Overall, empirical investigations and theoretical explanations clearly enlighten the relationships between MSA and procrastination. However, to the present authors' knowledge, no empirical study was addressed this relationship. Therefore, this study aimed to close this gap by determining whether there are any associations between MSA and procrastination among adolescents. In this regard, the relevant research hypothesis is below:

H3. There is a positive relationship between MSA and procrastination among adolescents.

\section{The Role of MSA in the Relationship Between Self-Control and Procrastination}

Further than determining whether there is a direct link between trait self-control and procrastination among adolescents, the current study's second main aim is to investigate how trait self-control and procrastination are linked indirectly through the alternate behaviors that procrastinators engage in instead of their planned event. Specifically, this study examined the role of MSA as a possible correlation between trait self-control and procrastination. In this context, previous researches has provided preliminary evidence on the mediator role of MSA in the relationship between self-control and procrastination (e.g., Çebi et al., 2019; Reinecke \& Hofmann, 2016; Yang et al., 2019). For example, Yang et al. (2019) revealed that problematic smartphone use plays a mediating role between self-regulation and academic procrastination among Chinese university students. Additionally, based on the strength model of self-control, Reinecke et al. (2014) proposed that ego-depleted people suffering from impaired state self-control are less likely to avoid hedonic pleasures. One of the most common desires 
of daily life is to use the media. Ego-depleted people have a greater inclination to give in to the temptation to use media, even though this desire interferes with other objectives such as completing work or managing other crucial activities, because of their decreased self-control ability. Similarly, in this study, we suggested that screen devices may stimulate desires by disrupting self-control and thereby lead to procrastination. In this regard, the relevant research hypothesis is below:

H4. MSA has a mediating role in the relationship between self-control and procrastination.

\section{Method}

This study aimed to examine and predict the relationship between the self-control, MSA, and procrastination behaviors of high school students, as well as to determine the mediating effect of MSA on the relationship between procrastination and self-control.

\section{Research Model and Hypotheses}

The research model was summed up in Fig. 1.

\section{Participants}

This study used the convenience sampling method to determine the participants. Convenience sampling is a nonprobability sampling method that allows the inclusion of participants who are eligible in terms of conditions such as voluntariness, accessibility, cost, and time (Etikan et al., 2016). Participants of the study consisted of 390 students, 296 females and 94 males, who continue their education in various high schools in Turkey. High school education in this country covers the grade range of 9-12. The average age of the participants was 15.6, and all grades participated in the study. During the COVID-19 pandemic period, 95.6\% of the participants stated that their screen time increased, and
$72 \%$ stated that the behavior of procrastinating their educational tasks increased. Thus, as shown in Table 1, it was seen that the average daily information and communication technologies (ICT) usage of the participants (PC, tablet PC and smartphone usage time, TV viewing time) is $9 \mathrm{H} 10 \mathrm{M}$.

\section{Instruments}

The General Procrastination Scale (GPS-9) is the short form of Lay's General Procrastination Scale (Lay, 1986) and developed by (Sirois et al., 2019). Composed of 9 items, the internal consistency coefficient of the GPS- 9 scale and the test-retest reliability value were reported as .89. In this study, the GPS-9 was used after obtaining permission from the authors and adapting it to Turkish. During the scale adaptation process, the translation equivalence was checked by a linguist who made scale development studies. After the necessary corrections were made, the 9-item scale form was sent to two researchers experienced in scale development studies for expert opinion. Following the expert review, the Turkish form was translated back into English. The linguist compared this translation form and the items in the original form in terms of meaning, and the final scale form was obtained. CFA was conducted to determine the convenience of the scale structure with the data collected within the scope of this study. According to the goodness of fit values (CMIN / $\mathrm{DF}=4.232, \mathrm{GFI}=.946, \mathrm{CFI}=.947$, $\mathrm{RMSEA}=.091$ ), the scale factor structure had an acceptable fit with the data. A high score on the GPS-9 scale indicates a high procrastination level. The lowest score on the scale can be 9, and the highest 45 points. Finally, in this study, we calculated the GPS-9 internal reliability coefficient Cronbach Alpha value as .87.

The Multi-Screen Addiction Scale (MSAS) developed by Sarttepeci (2021), is a 5 Likert-type and has 15 items consisting of 3 sub-dimensions. There are 4 items in "Excessive Screen Time (EST)", 8 items in "Compulsive Behavior (CB)", and 3 items in "Loss of Control (LoC)". The Cronbach Alpha internal consistency coefficient was reported for the MSAS scale and its sub-dimensions ranges between

Fig. 1 Research model

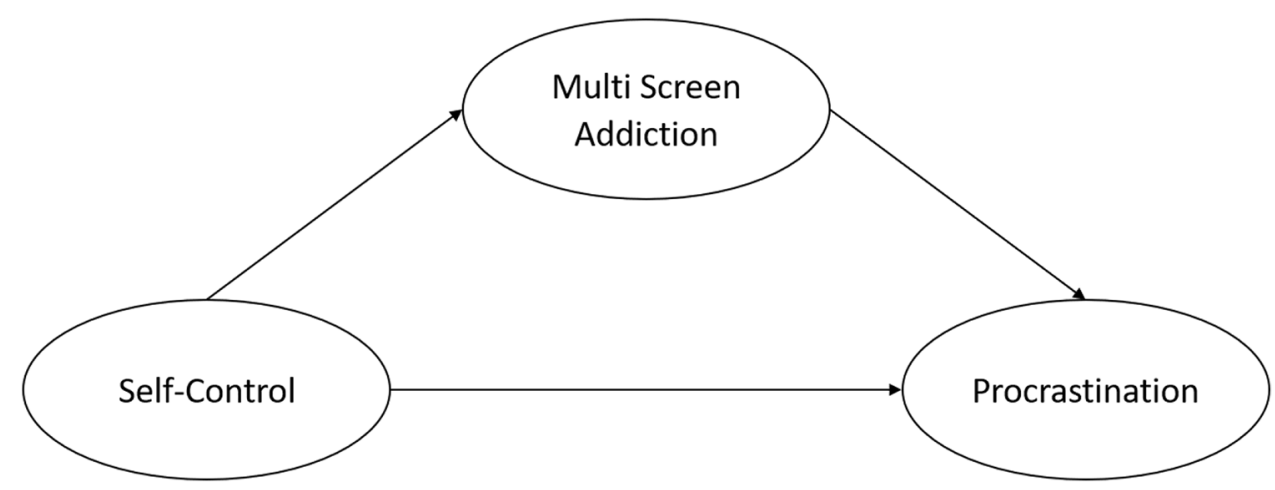


Table 1 Demographic characteristics and ICT usage status

\begin{tabular}{|c|c|c|c|}
\hline & & f & $\%$ \\
\hline \multirow[t]{2}{*}{ Gender } & Female & 296 & 75.9 \\
\hline & Male & 94 & 24.1 \\
\hline \multirow[t]{4}{*}{ Grade } & 1.00 & 121 & 31.0 \\
\hline & 2.00 & 127 & 32.6 \\
\hline & 3.00 & 102 & 26.2 \\
\hline & 4.00 & 40 & 10.3 \\
\hline \multirow{3}{*}{$\begin{array}{l}\text { Do you think that you spend more time with any screen (TV, Computer, Smartphone, Tablet, etc.) due to the Covid-19 } \\
\text { outbreak? }\end{array}$} & Yes & 319 & 81.8 \\
\hline & Partly & 54 & 13.8 \\
\hline & No & 17 & 4.4 \\
\hline \multirow{4}{*}{$\begin{array}{l}\text { Do you think that you have procrastinated your tasks (homework, attending live classes, etc.) more than before, due to the } \\
\text { Covid-19 outbreak? }\end{array}$} & Yes & 160 & 41.0 \\
\hline & Partly & 109 & 31.0 \\
\hline & No & 121 & 28.0 \\
\hline & Min & Max & M \\
\hline Age & 13.00 & 19.00 & 15.6 \\
\hline Daily TV viewing time (hours) & .00 & 7.00 & 1.28 \\
\hline Daily computer and tablet PC usage time (hours) & .00 & 16.00 & 3.39 \\
\hline Daily smartphone usage time (hours) & .00 & 17.00 & 4.50 \\
\hline
\end{tabular}

.71-92. CFA was conducted to examine the compatibility of the scale structure with the collected data. Accordingly, it was found that there was an acceptable fit between the factor structure of the MSAS scale and the data (CMIN / $\mathrm{DF}=4.232, \mathrm{GFI}=.946, \mathrm{CFI}=.947, \mathrm{RMSEA}=.091) . \mathrm{In}$ addition, we figured the MSAS and its sub-dimensions internal reliability coefficient value as between .70-.93.

Brief Self-Control Scale (BSCS) was developed by Tangney et al. (2004), and it is the short form of the Self-control Scale created by the same authors. There is a 5-factor structure in the long-form. Tangney et al. (2004) used the scale total score or the BSCS total score in analyzes, as there was a little change in the different analyzes performed in the correlations between these factors. While the SCS internal consistency coefficient Cronbach Alpha value was .89, the BSCS was reported as .83. In this study, the 13-items BSCS was used with permission from Nebioglu et al. (2012), which adapted this scale to Turkish. EFA and CFA were performed to reveal the factor structure of BSCS. EFA and CFA were applied to two separate data sets created by randomly split the total sample into two. Each split sample contained 148 males and 47 females. The factor structure of the BSCS scale was examined and EFA was applied. According to the EFA result, a single factor structure has emerged. The total variance explained by this single factor structure is $30.68 \%$ and item factor loadings alter between .42 and .72 CFA was performed to examine the compatibility of the single factor structure with the data. When the goodness of fit values were controlled $(\mathrm{CMIN} / \mathrm{DF}=2.247, \mathrm{GFI}=.922$, $\mathrm{CFI}=.892$, RMSEA $=.069$ ), it was found that the factor structure had an acceptable fit with the data. The scores of the participants were calculated by reverse scoring 9 items in the BSCS scale, as in the original form. The high score that the participants got from the scale indicates a high level of self-control. A minimum of 13 and a maximum of 65 scores can be obtained on the scale. We calculated the BCSC scale internal consistency Cronbach's alpha coefficient to be 79 (Appendix Figs. 5, 6, 7).

\section{Data Collection and Analysis}

The data set was collected online voluntarily with the data collection tool consisting of the participant consent form, personal information form, GPS-9, MSAS, and BSCS. If the participant approves the consent form, he/she can access other parts of the data collection tools. If he/she did not give his consent, his response was recorded without seeing the other parts of the data collection tool. The data collection phase covered the period from November 2020 to March 2021. Accordingly, 420 people answered the data collection tool, and 30 of them were excluded from the analysis process because they did not approve the participant consent form.

SPSS 27.0 was used to calculate descriptive statistics and AMOS 27.0 for path and mediation analysis. The skewness and kurtosis values of each of the items in the scales ranged from -1.5 to +1.5 (Tabachnick \& Fidell, 2007). Furthermore, the skewness and kurtosis values related to the distribution of the scores obtained from the overall scales found .231 and -.553 in procrastination, -.146 and -.207 in self-control, and .107 and - .718 in MSA. In addition, multivariate kurtosis critical ration was less than 10 (Kline, 2015). Accordingly, multivariate normality was ensured, and 
the maximum likelihood was used as the estimation method. The chi-square goodness of fit test, RMSEA, CFI, and GFI values were used for testing the measurement model consisting of self-control, MSA, and procrastination variables. When the measurement model was tested, it was found to be $\mathrm{CMIN} / \mathrm{DF}=1.99, \mathrm{RMSEA}=.05, \mathrm{GFI}=.89$, and $\mathrm{CFI}=.91$. Accordingly, it can say be said that the measurement model has an acceptable fit (Bentler, 1980; Tabachnick \& Fidell, 2007).

\section{Results}

Table 2 presents the descriptive statistics and correlation among the variables in the total sample, males, and females. It was found that there were no gender differences among the study variables. Findings showed females and males scored no significant difference in procrastination $(\mathrm{t}[388]=1.04$, $p>.01)$, self-control $(\mathrm{t}[388]=-1.00, p>.01)$, and MSA $(\mathrm{t}[388]=.85, p>.01)$.

In the total sample, the procrastination level of the participants was low $(M=23.60, S D=7.60)$. The $S C S$ score of the participants was 3.43 , and it was at a medium level. The MSA score was relatively low as 42.31 .
According to Table 2, in total sample, it was discerned that there is a medium level and negative $(r=-.59$, $p<0.01)$ relationship between procrastination and selfcontrol. Also there is a medium level and positive $(\mathrm{r}=.54$, $p<0.01)$ relationship among procrastination and MSA. In addition, there is a negative and medium level relationship between self-control and MSA $(r=-.59, p<0.01)$. Additionally, similar correlation patterns were detected between males and females.

\section{Latent Variable Path Model}

Initially, the model examining the relationship between self-control and procrastination was created and the $\mathrm{H} 1$ hypothesis was tested. According to the goodness of fit values presented in Fig. 2, it was discerned that the model has a good data fit. In addition, it was identified that selfcontrol was a significant predictor of procrastination $\left(\beta=-.70, R^{2}=.49\right)$, and the $\mathrm{H} 1$ hypothesis was accepted.

As in Fig. 3, a model was constructed to identify the relationship between self-control and MSA. As shown in Fig. 2, the model has a good data fit. There is a statistically significant negative relationship between self-control and MSA ( $\beta=-.79, \mathrm{R}^{2}=.63$ ). Accordingly, MSA is a significant predictor of procrastination, and the $\mathrm{H} 2$ hypothesis was accepted.
Table 2 Descriptive statistics and correlations of self-control, MSA, and procrastination between males, females, and the total sample
Fig. 2 Path coefficient between self-control and procrastination

\begin{tabular}{lllllll}
\hline Variables & $\mathrm{M}$ & Mean/Items & $\mathrm{SD}$ & 1 & 2 & 3 \\
\hline 1- Procrastination & 23.60 & 2.62 & 7.60 & 1 & & \\
2- Self-control & 44.57 & 3.43 & 8.58 & $-.59^{* *}$ & 1 & \\
3- MSA & 42.31 & 2.82 & 13.16 & $.54^{* *}$ & $-.59^{* *}$ & 1 \\
Females (n=296) & & & & & & \\
1- Procrastination & 23.83 & 2.65 & 7.66 & 1 & & \\
2- Self-control & 44.32 & 3.41 & 8.67 & $-.56^{* *}$ & 1 & $-.57^{* *}$ \\
3- MSA & 42.63 & 2.84 & 13.23 & $.54^{* *}$ & & \\
Males (n=94) & & & & & & \\
1- Procrastination & 22.89 & 2.54 & 7.41 & 1 & & \\
2- Self-control & 45.34 & 3.49 & 8.27 & $-.70^{* *}$ & 1 & \\
3- MSA & 41.31 & 2.75 & 12.96 & $.55^{* *}$ & $-.67^{* *}$ & 1 \\
\hline
\end{tabular}

**Correlation is significant at the 0.01 level (2-tailed)

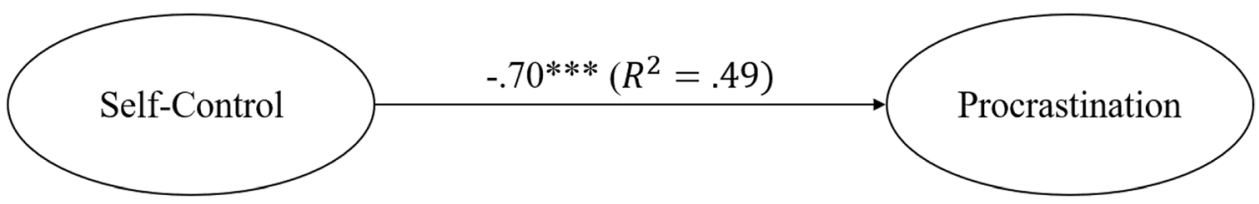

$\mathrm{CMIN} / \mathrm{DF}=2.506, \mathrm{GFI}=.919, \mathrm{CFI}=.918, \mathrm{RMSEA}=.062$

$* * * p<.001$ 

MSA and procrastination
Fig. 3 Path coefficient between

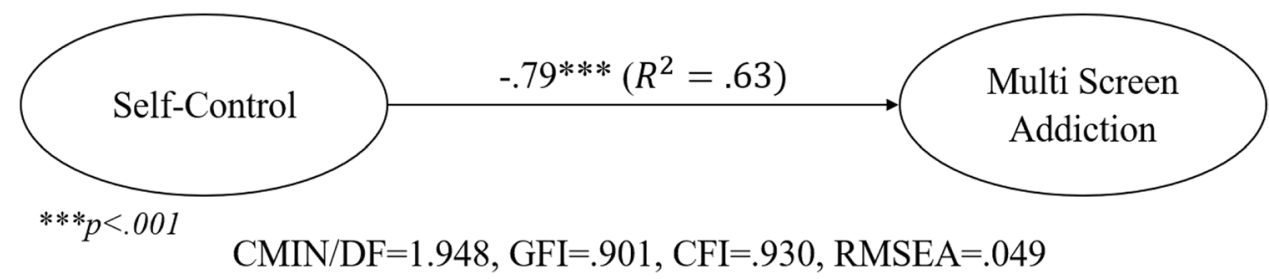

Fig. 4 Mediating effect

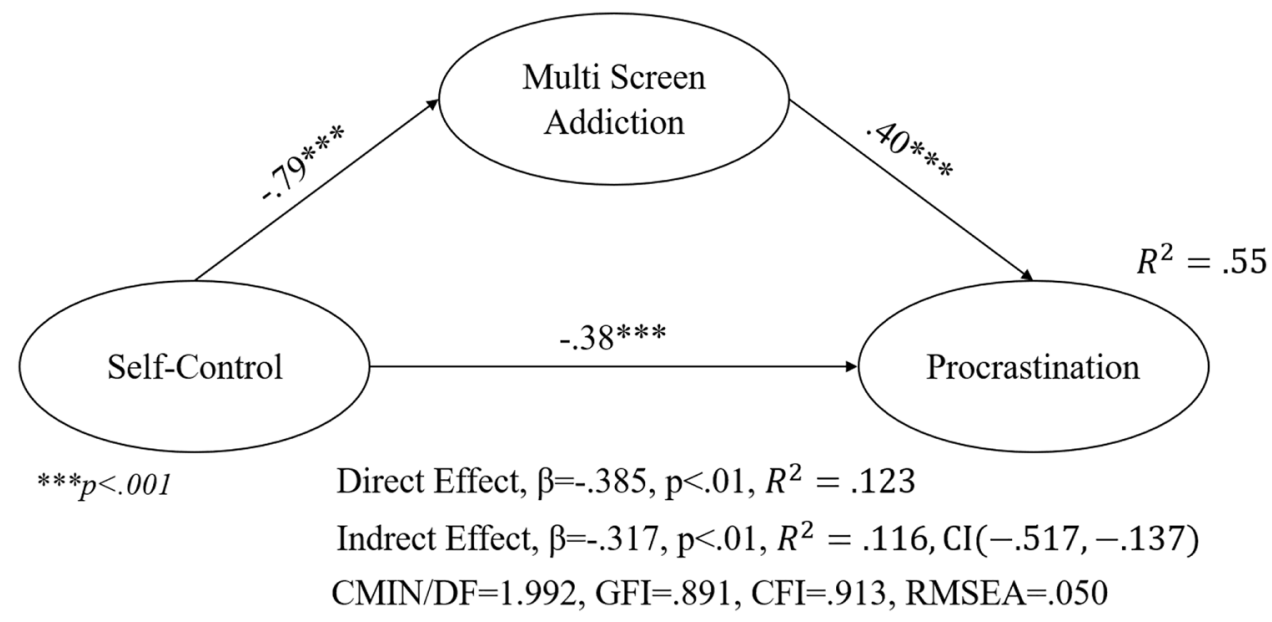

\section{Mediating Effect}

To determine the mediating effect of MSA on the relationship between self-control and procrastination, the model presented in Fig. 4 was established and it was obtained as an acceptable data-model fit. As shown in Fig. 3, the direct effect of the mediator variable MSA on procrastination was significant. In this case, the $\mathrm{H} 3$ hypothesis was accepted. The direct effect of self-control and procrastination $(\beta=-.38$, $p<.001$ ) was devised as significant when the MSA mediator variable was included in the model. The mediator variable caused the coefficient of connection between self-control and procrastination to decrease. According to the findings from the Bootstrap analysis, the indirect effect of self-control on procrastination via MSA is significant $(\beta=-.317$, CI $[-.517,-.137], p<.001)$. Pursuant, the mediating effect of MSA on the relationship between self-control and procrastination is significant and the $\mathrm{H} 4$ hypothesis was accepted.

\section{Discussion}

The study aimed to examine the relationships among adolescents' levels of self-control, MSA, and procrastination. MSA is a relatively new concept and its importance has become evident especially during the pandemic period. For this reason, the studies on problematic use of technology were examined and the modeling and testing of four hypotheses
Table 3 Hypothesis testing results

\begin{tabular}{lll}
\hline Hypothesis & Accept/Reject \\
\hline H1 & Self-control $\rightarrow$ Procrastination & Accept \\
H2 & Self-control $\rightarrow$ MSA & Accept \\
H3 & MSA $\rightarrow$ Procrastination & Accept \\
H4 & Self-control $\rightarrow$ MSA $\rightarrow$ Procrastination & Accept \\
\hline
\end{tabular}

were realized. According to the results, all hypotheses were supported (see Table 3).

According to the findings of this study, there is a moderate negative relationship between procrastination and selfcontrol, and a moderately positive relationship with MSA. In addition, there is a negative and medium level relationship between self-control and MSA. Self-control can be considered an important variable to prevent problematic technology use behaviors. Self-control includes an active and constructive control process in terms of the individual's emotions, thoughts, behavior, and motivation. On the other hand, procrastination is the tendency to postpone a task or to avoid a task that has negative effects on the individual (Tuckman, 1998). Therefore, the results can be understood when examined in the context of the operational definitions of the concepts. Procrastination behavior, as a multidimensional concept, includes difficulties in maintaining self-control and not being able to manage time. In the study by Yildız Durak (2020), adolescents with high self-regulation skills were less likely to become social media addicts. Lack of self-control 
and procrastination can also lead to problematic use of digital technologies.

There is a statistically significant negative relationship between self-control and procrastination (H1). In the study conducted by Kim et al. (2017), it was found that self-control behavior directly affects procrastination, and the relationship between self-control and procrastination affects Internet addiction. Indeed, Steel (2007) argued that procrastination is a failure of self-regulation. In this context, the findings of the present study coincide with the results in the literature (e.g., Geng et al., 2018; Kim et al., 2017; Steel, 2007; Zhao et al., 2019).

There is a statistically significant negative relationship between self-control and MSA (H2). In addition, the direct effect of the mediator variable, MSA, between procrastination is significant (H3). According to Van Deursen et al. (2015), the basis of addictive behaviors is the loss of control of individuals. Similarly, according to Zhang et al. (2019), those who procrastinate have difficulty resisting their impulses. In this context, in accordance with the research results, Y1ldiz Durak (2020) stated that adolescents' selfcontrol skills were effective in using technologies such as the internet and social media for purposes and in managing time. In this case, addictive behaviors may cause a decrease in academic performance (Y1ldız Durak, 2019).

The mediating effect of multiple screen addiction on the relationship between self-control and procrastination is important (H4). The results of the study by Zhao et al. (2019) showed that self-control has an inhibiting effect on procrastination tendency. In the study by Yildiz Durak (2018), it was found that nomophobic behaviors and addictive behaviors have a positive relationship with adolescents' locus of control. Yang et al. (2019) revealed that problematic smartphone use plays a mediating role between selfregulation and academic procrastination. In this context, literature reviews provide preliminary findings that support the research results. As a matter of fact, one of the most common desires of daily life in today's societies is to use the media (Durak \& Saritepeci, 2019). The COVID-19 pandemic process has also made this desire clear. Therefore, in the present work, the mediating effect of MSA on the relationship between self-control and procrastination increased with the pandemic process.

\section{Limitations and Recommendations}

There are some limitations to this study. The results of this research examine the relationship between self-control, MSA, and procrastination. Therefore, a causal relationship between variables cannot be inferred. In the current study, the relationships between self-control, MSA, and procrastination were determined using a cross-sectional research design. However, the research design does not reveal cause-effect relationships. Results can be studied with similar groups in different cultures and differentiation can be examined. In the study, the data were collected using self-report methods. This situation carries the risk of biased answers. Then, the data were collected anonymously to reduce this risk. Triangulation, repeated measurements, and non-self-reporting measurements can be made in future studies. Using multiple approaches in future studies may strengthen the generalizability and validity of the results of this study. The current study has a cross-sectional research design. Longitudinal and qualitative studies can be carried out in future studies. Also, MSA is a multidimensional structure. Future studies may apply the proposed model according to MSA dimensions, especially in academic circles.

According to the research results, supporting the selfcontrol tendency can strengthen its negative effect on the tendency to procrastinate. For this reason, software that supports self-control behavior by providing locus of control can be used especially in academic contexts. The role of this software can be explored through empirical study to discover the effect of self-control on procrastination. On the other hand, MSA behaviors and qualitative personality studies can be done that can make people procrastinate and how they can avoid it, and personalize interventions for the individual. On the other hand, qualitative personality studies that also examine MSA behaviors towards how people can get rid of procrastination behavior can be conducted and personalized interventions can be made. In addition to these limitations, despite there is overwhelming evidence in favor of ego depletion research, it should be remembered that the ego-depletion theoretical model (self-control failure-ego depletion effect) has come under heavy criticism (see Friese et al., 2019).

Supplementary Information The online version contains supplementary material available at https://doi.org/10.1007/s12144-021-02472-2.

Data Availability The datasets created during and/or analyzed during the current study are available from the corresponding author on reasonable request.

\section{Declarations}

Ethical Approval The study was in accordance with the ethical standards of the institutional and/or national research committee and with the 1964 Helsinki declaration and its later amendments or comparable ethical standards.

Informed Consent Informed consent was obtained from all individual. participants included in the study.

Conflict of Interest The authors declare that they have no conflict of interest. 


\section{References}

Allemand, M., Job, V., \& Mroczek, D. K. (2019). Self-control development in adolescence predicts love and work in adulthood. Journal of Personality and Social Psychology, 117(3), 621-634. https://doi.org/10.1037/pspp0000229

American Psychiatric Association. (2013). Diagnostic and statistical manual for mental disorders (5th ed.). American Psychiatric Association.

Andreassen, C. S. (2015). Online social network site addiction: A comprehensive review. Current Addiction Reports, 2, 175-184.

Andreassen, C. S., Billieux, J., Griffiths, M. D., Kuss, D. J., Demetrovics, Z., Mazzoni, E., \& Pallesen, S. (2016). The relationship between addictive use of social media and video games and symptoms of psychiatric disorders: A large-scale cross-sectional study. Psychology of Addictive Behaviors, 30(2), 252-262. https://doi.org/10.1037/adb0000160

Balhara, Y. P. S., Verma, K., \& Bhargava, R. (2018). Screen time and screen addiction: Beyond gaming, social media and pornography-a case report. Asian Journal of Psychiatry, 35, 77-78.

Baumeister, R. F., \& Alquist, J. L. (2009). Is there a downside to good self-control? Self and Identity, 8, 115-130.

Baumeister, R. F., \& Tierney, J. (2011). Willpower: Rediscovering the greatest human strength. Penguin.

Baumeister, R. F., Bratslavsky, E., Muraven, M., \& Tice, D. M. (1998). Ego depletion: Is the active self a limited resource? Journal of Personality and Social Psychology, 74(5), 1252.

Baumeister, R. F., Vohs, K. D., \& Tice, D. M. (2007). The strength model of self-control. Current Directions in Psychological Science, 16, 351-355.

Bentler, P. M. (1980). Multivariate analysis with latent variables: Causal modeling. Annual Review of Psychology, 31(1), 419-456.

Çebi, A., Reisoğlu, İ., \& Bahçekapılı, T. (2019). The relationships among academic procrastination, self-control, and problematic mobile use: Considering the differences over personalities. Addicta: The Turkish Journal on Addictions, 6, 447-468. https://doi.org/10.15805/addicta.2019.6.3.0082

Chang, F.-C., Chiu, C.-H., Chen, P.-H., Miao, N.-F., Chiang, J.-T., \& Chuang, H.-Y. (2018). Computer/mobile device screen time of children and their eye care behavior: The roles of risk perception and parenting. Cyberpsychology, Behavior and Social Networking, 21(3), 179-186.

de Ridder, D. T., de Boer, B. J., Lugtig, P., Bakker, A. B., \& van Hooft, E. A. (2011). Not doing bad things is not equivalent to doing the right thing: Distinguishing between inhibitory and initiatory self-control. Personality and Individual Differences, 50(7), 1006-1011. https://doi.org/10.1016/j.paid.2011.01.015

de Ridder, D. T. D., Lensvelt-Mulders, G., Finkenauer, C., Stok, M., \& Baumeister, R. F. (2012). Taking stock of self-control: A meta-analysis of how trait self-control relates to a wide range of behaviors. Personality and Social Psychology Review, 16, 76-99. https://doi.org/10.1177/1088868311418749

Durak, H. Y., \& Saritepeci, M. (2019). Modeling the effect of new media literacy levels and social media usage status on problematic internet usage behaviours among high school students. Education and Information Technologies, 24(4), 2205-2223.

Ent, M. R., Baumeister, R. F., \& Tice, D. M. (2015). Trait self-control and the avoidance of temptation. Personality and Individual Differences, 74, 12-15. https://doi.org/10.1016/j.paid.2014.09.031

Etikan, I., Musa, S. A., \& Alkassim, R. S. (2016). Comparison of convenience sampling and purposive sampling. American Journal of Theoretical and Applied Statistics, 5(1), 1-4.

Ferrari, J. R., \& Emmons, R. A. (1995). Methods of procrastination and their relation to self-control and self-reinforcement: An exploratory study. Journal of Social Behavior and Personality, 10(1), 135-142.

Friese, M., Loschelder, D. D., Gieseler, K., Frankenbach, J., \& Inzlicht, M. (2019). Is ego depletion real? An analysis of arguments. Personality and Social Psychology Review, 23(2), 107-131.

Geng, J., Han, L., Gao, F., Jou, M., \& Huang, C. C. (2018). Internet addiction and procrastination among Chinese young adults: A moderated mediation model. Computers in Human Behavior, 84, 320-333. https://doi.org/10.1016/j.chb.2018.03.013

Gottfredson, M. R., \& Hirschi, T. (1990). A general theory of crime. Stanford University Press.

Grasmick, H. G., Tittle, C. R., Bursik, R. J. J., \& Arneklev, B. J. (1993). Testing the core empirical implications of Gottfredson and Hirschi's general theory of crime. Journal of Research in Crime and Delinquency, 30, 5-29. https://doi.org/10.1177/00224 27893030001002

Hagger, M. S., Wood, C., Stiff, C., \& Chatzisarantis, N. L. (2010). Ego depletion and the strength model of self-control: A meta-analysis. Psychological Bulletin, 136(4), 495-525. https://doi.org/10.1037/ a0019486

Haug, S., Castro, R. P., Kwon, M., Filler, A., Kowatsch, T., \& Schaub, M. P. (2015). Smartphone use and smartphone addiction among young people in Switzerland. Journal of Behavioral Addictions, 4(4), 299-307. https://doi.org/10.1556/2006.4.2015.037

Hofmann, W., Reinecke, L., \& Meier, A. (2017). Of sweet temptations and bitter aftertaste: Self-control as a moderator of the effects of media use on well-being. In L. Reinecke \& M. B. Oliver (Eds.), The Routledge handbook of media use and well-being: International perspectives on theory and research on positive media effects (pp. 211-222). Routledge.

Inzlicht, M., Legault, L., \& Teper, R. (2014). Exploring the mechanisms of self-control improvement. Current Directions in Psychological Science, 23(4), 302-307. https://doi.org/10.1177/09637 21414534256

Job, V., Dweck, C. S., \& Walton, G. M. (2010). Ego depletion-is it all in your head? Implicit theories about willpower affect selfregulation. Psychological Science, 21(11), 1686-1693. https://doi. org/10.1177/0956797610384745

Kadzikowska-Wrzosek, R. (2020). Insufficient sleep among adolescents: The role of bedtime procrastination, chronotype and autonomous vs. controlled motivational regulations. Current Psychology, 39, 1031-1040. https://doi.org/10.1007/s12144-018-9825-7

Kamphorst, B. A., Nauts, S., De Ridder, D. T., \& Anderson, J. H. (2018). Too depleted to turn in: The relevance of end-of-the-day resource depletion for reducing bedtime procrastination. Frontiers in Psychology, 9, 252. https://doi.org/10.3389/fpsyg.2018.00252

Kim, K. R., \& Seo, E. H. (2015). The relationship between procrastination and academic performance: A meta-analysis. Personality and Individual Differences, 82, 26-33. https://doi.org/10.1016/j. paid.2015.02.038

Kim, J., Hong, H., Lee, J., \& Hyun, M. H. (2017). Effects of time perspective and self-control on procrastination and internet addiction. Journal of Behavioral Addictions, 6(2), 229-236. https://doi.org/ 10.1556/2006.6.2017.017

Klassen, R. M., Ang, R. P., Chong, W. H., Krawchuk, L. L., Huan, V. S., Wong, I. Y., \& Yeo, L. S. (2009). A cross-cultural study of adolescent procrastination. Journal of Research on Adolescence, 19(4), 799-811. https://doi.org/10.1111/j.1532-7795.2009. 00620. $\mathrm{x}$

Kline, R. B. (2015). Principles and practice of structural equation modeling. Guilford Publications.

Klingsieck, K. B. (2013). Procrastination: When good things don't come to those who wait. European Psychologist, 18, 24-34. https://doi.org/10.1027/1016-9040/a000138

Kuhnle, C., Hofer, M., \& Kilian, B. (2011). The relationship of selfcontrol, procrastination, motivational interference and regret 
with school grades and life balance. Diskurs Kindheits-und Jugendforschung/Discourse. Journal of Childhood and Adolescence Research, 6(1), 9-10.

Lavoie, J., \& Pychyl, T. A. (2001). Cyber-slacking and the procrastination super- highway: A web-based survey of online procrastination, attitudes, and emotion. Social Science Computer Review, 19, 431e444. https://doi.org/10.1177/089443930101900403

Lay, C. H. (1986). At last, my research article on procrastination. Journal of Research in Personality, 20(4), 474-495.

Lin, T. T. C., Kononova, A., \& Chiang, Y. H. (2019). Screen addiction and media multitasking among American and Taiwanese users. Journal of Computer Information Systems, 60(6), 583-592. https://doi.org/10.1080/08874417.2018.1556133

Macit, Z. B., \& Kavafoğlu, S. (2019). Screen: Subject of all information technology addiction. Middle Black Sea Journal of Health Science, 5(3), 293-301. https://doi.org/10.19127/mbsjohs.542122.

Mehroof, M., \& Griffiths, M. D. (2010). Online gaming addiction: The role of sensation seeking, self-control, neuroticism, aggression, state anxiety, and trait anxiety. Cyberpsychology, Behavior, and Social Networking, 13(3), 313-316. https://doi.org/10.1089/ cyber.2009.0229

Metcalfe, J., \& Mischel, W. (1999). A hot/cool system analysis of delay of gratification: Dynamics of willpower. Psychological Review, 106, 3-19.

Mischel, W., \& Ebbesen, E. B. (1970). Attention in delay of gratification. Journal of Personality and Social Psychology, 16(2), 329-337.

Monacis, L., Griffiths, M. D., Limone, P., \& Sinatra, M. (2021). The risk of social media addiction between the ideal/false and true self: Testing a path model through the tripartite person-centered perspective of authenticity. Telematics and Informatics, 65, 101709. https://doi.org/10.1016/j.tele.2021.101709

Muraven, M., \& Baumeister, R. F. (2000). Self-regulation and depletion of limited resources: Does self-control resemble a muscle? Psychological Bulletin, 126, 247-259. https://doi.org/10.1037/ 0033-2909.126.2.247

Nebioglu, M., Konuk, N., Akbaba, S., \& Eroglu, Y. (2012). The investigation of validity and reliability of the Turkish version of the brief self-control scale. Klinik Psikofarmakoloji Bülteni-Bulletin of Clinical Psychopharmacology, 22(4), 340-351.

Pratt, T. C., \& Cullen, F. T. (2000). The empirical status of Gottfredson and Hirschi's general theory of crime: A meta-analysis. Criminology, 38(3), 931-964.

Rebetez, M. M. L., Rochat, L., Barsics, C., \& Van der Linden, M. (2016). Procrastination as a self-regulation failure: The role of inhibition, negative affect, and gender. Personality and Individual Differences, 101, 435-439. https://doi.org/10.1016/j.paid.2016. 06.049

Reinecke, L., \& Hofmann, W. (2016). Slacking off or winding down? An experience sampling study on the drivers and consequences of media use for recovery versus procrastination. Human Communication Research, 42(3), 441-461. https://doi.org/10.1111/ hcre. 12082

Reinecke, L., Hartmann, T., \& Eden, A. (2014). The guilty couch potato: The role of ego depletion in reducing recovery through media use. Journal of Communication, 64(4), 569-589. https:// doi.org/10.1111/jcom.12107

Reinecke, L., Meier, A., Beutel, M. E., Schemer, C., Stark, B., Wölfling, K., \& Müller, K. W. (2018). The relationship between trait procrastination, internet use, and psychological functioning: Results from a community sample of German adolescents. Frontiers in Psychology, 9, 913. https://doi.org/10.3389/fpsyg.2018. 00913

Ronen, T., Hamama, L., Rosenbaum, M., \& Mishely-Yarlap, A. (2016). Subjective well-being in adolescence: The role of selfcontrol, social support, age, gender, and familial crisis. Journal of Happiness Studies, 17(1), 81-104. https://doi.org/10.1007/ s10902-014-9585-5

Saritepeci, M. (2020). Predictors of cyberloafing among high school students: Unauthorized access to school network, metacognitive awareness and smartphone addiction. Education and Information Technologies, 25, 2201-2219. https://doi.org/10.1007/ s10639-019-10042-0

Saritepeci, M. (2021). Multiple screen addiction scale: Validity and reliability study. Instructional Technology and Lifelong Learning, 2(1), 1-17.

Seneecal, C., Lavoie, K., \& Koestner, R. (1997). Trait and situational factors in pro- crastination: An interactional model. Journal of Social Behavior and Personality, 12, 889e903.

Şimşir, Z., \& Dilmaç, B. (2021). The mediating roles of grit and life satisfaction in the relationship between self-discipline and peace: Development of the self-discipline scale. Current Psychology. Advance online publication. 10.1007/s12144-021-01515-y.

Sirois, F., \& Pychyl, T. (2013). Procrastination and the priority of shortterm mood regulation: Consequences for future self. Social and Personality Psychology Compass, 7(2), 115-127. https://doi.org/ $10.1111 / \mathrm{spc} 3.12011$

Sirois, F. M., Yang, S., \& van Eerde, W. (2019). Development and validation of the General Procrastination Scale (GPS-9): A short and reliable measure of trait procrastination. Personality and Individual Differences, 146, 26-33. https://doi.org/10.1016/j. paid.2019.03.039

Steel, P. (2007). The nature of procrastination. Psychological Bulletin, 133(1), 65-94.

Tabachnick, B. G., \& Fidell, L. S. (2007). Using multivariate statistics (6th ed.). Pearson.

Tang, J., Yu, Y., Du, Y., Ma, Y., Zhang, D., \& Wang, J. (2014). Prevalence of internet addiction and its association with stressful life events and psychological symptoms among adolescent internet users. Addictive Behaviors, 39(3), 744-747. https://doi.org/10. 1016/j.addbeh.2013.12.010

Tangney, J., Baumeister, R. F., \& Boone, A. L. (2004). High self-control predicts good adjustment, less pathology, better grades, and interpersonal success. Journal of Personality, 72, 271-324.

Tuckman, B. W. (1998). Using tests as an incentive to motivate procrastinators to study. The Journal of Experimental Education, 66(2), 141-147.

Vainik, U., García-García, I., \& Dagher, A. (2019). Uncontrolled eating: A unifying heritable trait linked with obesity, overeating, personality and the brain. European Journal of Neuroscience, 50(3), 2430-2445. https://doi.org/10.1111/ejn.14352

Van Deursen, A. J., Bolle, C. L., Hegner, S. M., \& Kommers, P. A. (2015). Modeling habitual and addictive smartphone behavior: The role of smartphone usage types, emotional intelligence, social stress, self-regulation, age, and gender. Computers in Human Behavior, 45, 411-420. https://doi.org/10.1016/j.chb.2014.12.039

Vazsonyi, A. T., Machackova, H., Sevcikova, A., Smahel, D., \& Cerna, A. (2012). Cyberbullying in context: Direct and indirect effects by low self-control across 25 European countries. European Journal of Developmental Psychology, 9(2), 210-227. https://doi.org/10. 1080/17405629.2011.644919

Wang, C. W., Chan, C. L., Mak, K. K., Ho, S. Y., Wong, P. W., \& Ho, R. T. (2014). Prevalence and correlates of video and internet gaming addiction among Hong Kong adolescents: A pilot study. The Scientific World Journal, 874648. https://doi.org/10.1155/2014/ 874648

Yang, Z., Asbury, K., \& Griffiths, M. D. (2019). An exploration of problematic smartphone use among Chinese university students: Associations with academic anxiety, academic procrastination, self-regulation and subjective wellbeing. International Journal of Mental Health and Addiction, 17(3), 596-614. https://doi.org/ 10.1007/s11469-018-9961-1 
Yildız Durak, H. (2018). What would you do without your smartphone? Adolescents' social media usage, locus of control, and loneliness as a predictor of nomophobia. Addicta: The Turkish Journal on Addictions, 5(3), 543-557. https://doi.org/10.15805/addicta. 2018.5.2.0025

Yildız Durak, H. (2019). Investigation of nomophobia and smartphone addiction predictors among adolescents in Turkey: Demographic variables and academic performance. The Social Science Journal, 56(4), 492-517. https://doi.org/10.1016/j.soscij.2018.09.003

Yıldız Durak, H. (2020). Modeling of variables related to problematic internet usage and problematic social media usage in adolescents. Current Psychology, 39, 1375-1387. https://doi.org/10.1007/ s12144-018-9840-8

Zhang, S., Liu, P., \& Feng, T. (2019). To do it now or later: The cognitive mechanisms and neural substrates underlying procrastination.
Wiley Interdisciplinary Reviews: Cognitive Science, 10(4), e1492. https://doi.org/10.1002/wcs.1492

Zhao, J., Meng, G., Sun, Y., Xu, Y., Geng, J., \& Han, L. (2019). The relationship between self-control and procrastination based on the self-regulation theory perspective: The moderated mediation model. Current Psychology, Advance online publication. https:// doi.org/10.1007/s12144-019-00442-3

Author order reflects the weight of these contributions.

Publisher's note Springer Nature remains neutral with regard to jurisdictional claims in published maps and institutional affiliations. 